\title{
Strong laser polarization control of coherent phonon excitation in van der Waals material $\mathrm{Fe}_{3} \mathrm{GeTe}_{2}$
}

Yu Gong $\mathbb{D}^{1 凶}$, Ming Hu${ }^{2 凶}$, Nico Harris ${ }^{1}$, Zhonghua Yang ${ }^{2,3}, \mathrm{Ti} \mathrm{Xie}^{4}$, Alem Teklu ${ }^{1}$, Narayanan Kuthirummal ${ }^{1}$, Jacob koenemann ${ }^{1}$, Xianghan Xu $\mathbb{i D}^{5}$, Sang-Wook Cheong ${ }^{5}$, Will McLoud ${ }^{1}$ and Cheng Gong $\mathbb{D}^{4 凶}$

Optical manipulation of coherent phonon frequency in two-dimensional (2D) materials could advance the development of ultrafast phononics in atomic-thin platforms. However, conventional approaches for such control are limited to doping, strain, structural or thermal engineering. Here, we report the experimental observation of strong laser-polarization control of coherent phonon frequency through time-resolved pump-probe spectroscopic study of van der Waals (vdW) materials $\mathrm{Fe}_{3} \mathrm{GeTe}_{2}$. When the polarization of the pumping laser with tilted incidence is swept between in-plane and out-of-plane orientations, the frequencies of excited phonons can be monotonically tuned by as large as $3 \%(\sim 100 \mathrm{GHz})$. Our first-principles calculations suggest the strong planar and vertical inter-atomic interaction asymmetry in layered materials accounts for the observed polarization-dependent phonon frequencies, as in-plane/out-of-plane polarization modifies the restoring force of the lattice vibration differently. Our work provides insightful understanding of the coherent phonon dynamics in layered vdW materials and opens up new avenues to optically manipulating coherent phonons.

npj 2D Materials and Applications (2022)6:9; https://doi.org/10.1038/s41699-021-00275-4

\section{INTRODUCTION}

Coherent phonon is the quantized in-phase lattice vibration in solids. It is a fundamental medium to explore various quantum systems, including quantum dots ${ }^{1,2}$, superconducting qubits ${ }^{3-5}$, and integrated photonic devices ${ }^{6-9}$. Coherent phonon could easily reach terahertz $(\mathrm{THz})$ regime and become the basis for paradigm shift in various fields including high-frequency electronics ${ }^{10,11}$, information technology ${ }^{12,13}$, and medical diagnostics ${ }^{14}$. $\mathrm{THz}$ phonons could potentially reduce the footprint of phononic devices, improve spatial resolution of imaging techniques, and boost the speed of acousto-electronic/optical devices by several orders of magnitude compared to traditional ultrasonics in $\mathrm{MHz}$ frequencies.

Underlying all the aforementioned prospects, the ability to control coherent phonon frequency has far-reaching significance $^{15}$, especially in the emergent two-dimensional (2D) materials where the atomic layer thickness allows highly designable and manipulable inter-atomic interactions for phonon control. However, the phonon frequency control in 2D materials has thus far only been realized via doping, strain, structural and thermal engineering. For example, the phonon frequency can be controlled over $500 \mathrm{GHz}$ in graphene by doping ${ }^{16,17}$. The softening of phonon with increased strain has been observed in $\mathrm{MoS}_{2}{ }^{18,19}$, and the phonon modes $\mathrm{E}_{2 \mathrm{~g}}^{1}$ and $\mathrm{A}_{1 \mathrm{~g}}$ in $\mathrm{MoS}_{2}$ can be also shifted by adjusting the thickness (i.e., layer number) ${ }^{20,21}$ or by changing the temperature 22,23 . These approaches are either slow or irreversible.

In layered vdW materials, the atoms are bound with strong covalent bonds inside each constituent layer, while the adjacent layers are held together by weak vdW force. This unique structural feature generates strongly anisotropic physical properties. Hence, anisotropic electron, phonon and magnon coupling as well as their transport properties may be achieved in vdW materials. For example, the manipulation of thermal conductivity may be realized by exploring the phonon transport across a vdW interface ${ }^{24-27}$. The interaction between coherent phonons and charge carriers could lead to new paths for the generation of microwave frequencies, as the cases that have been explored for nanoelectromechanical systems in the $\mathrm{MHz}-\mathrm{GHz}$ frequency regime ${ }^{28}$, for piezoelectric heterostructures in the sub- $\mathrm{THz}$ regime ${ }^{29}$, and for semiconductor devices such as Schottky diodes $^{11,30}$ as well as tunneling devices ${ }^{10}$. Because of strong elastic anisotropy and phonon quantization, layered vdW materials could be ideal platforms for phonon nanoscopy ${ }^{31}$ and nondestructive imaging for molecules and cells coupled to vdW layers.

Therefore, understanding and manipulation of phonon frequency become increasingly attractive in vdW materials including graphene, transition metal dichalchogenides (e.g., $\mathrm{MoS}_{2}$ and $\mathrm{WS}_{2}$ ), metal chalcogenides (e.g., InSe and $\mathrm{GaSe}$ ), hexagonal boron nitride $(h-\mathrm{BN})$, to name a few ${ }^{32}$. However, although structural and temperature/thermal engineering of phonon frequency have been achieved in vdW materials, these approaches are not suitable for high speed/frequency microscopic phononic applications. It is desirable to have non-thermal and swift control of phonon frequency in layered vdW materials. So far vdW materials have shown a wide variety of fascinating physical phenomena. Take $\mathrm{Fe}_{3} \mathrm{GeTe}_{2}$ as an example, it has shown 2D itinerant ferromagnetism $^{33-36}$, topological nodal lines ${ }^{37}$, tunneling spin-valve behavior $^{38}$, heavy fermion states ${ }^{39}$, manipulable magnetic domains ${ }^{40,41}$, and strong electron correlation effects ${ }^{42}$. However, the nonthermal control of coherent phonon frequency has not been accomplished in vdW materials to the best of our knowledge.

\footnotetext{
${ }^{1}$ Department of Physics and Astronomy, College of Charleston, 58 Coming Street, Charleston, SC 29424, USA. ${ }^{2}$ Department of Mechanical Engineering, University of South Carolina, 541 Main Street, Columbia, SC 29208, USA. ${ }^{3}$ College of Architecture and Civil Engineering, Shenyang University of Technology, 110870 Shenyang, China. ${ }^{4}$ Department of Electrical and Computer Engineering and Quantum Technology Center, University of Maryland, College Park, MD 20742, USA. ${ }^{5}$ Rutgers Center for Emergent Materials and Department of Physics and Astronomy, Rutgers, The State University of New Jersey, Piscataway, NJ 08854, USA. 『email: gongy@cofc.edu; hu@sc.edu; gongc@umd.edu
} 


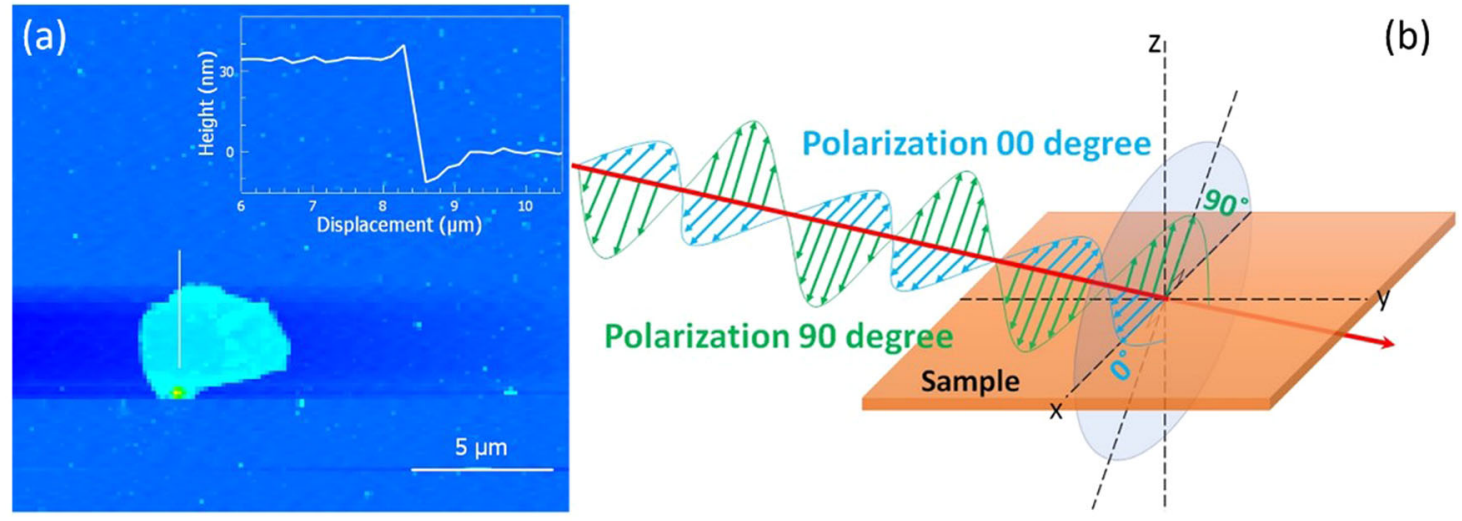

(b)
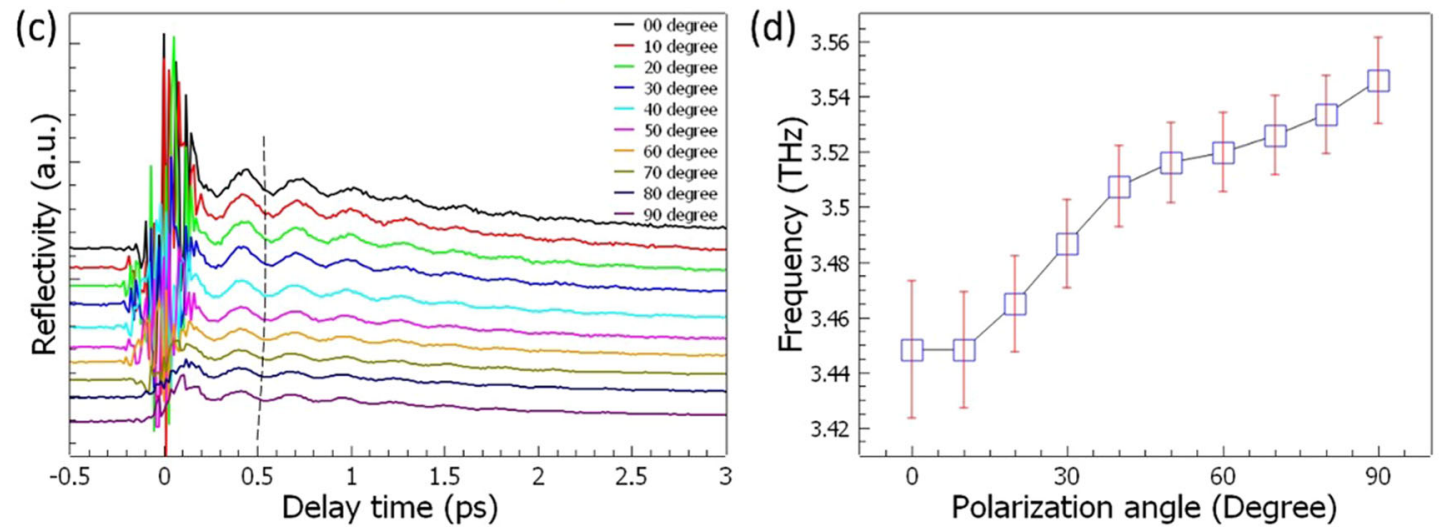

Fig. 1 Characterization of coherent phonon in $\mathrm{Fe}_{3} \mathrm{GeTe}_{2}$ flake. a AFM image of an exfoliated $\mathrm{Fe}_{3} \mathrm{GeTe}_{2}$ flake. The inset in Fig. 1 (a) is a height profile along the line cut indicated by white solid line on the flake. $\mathbf{b}$ An illustrated scheme of pump beam configuration. The pump beam (red solid line) is incident about $70^{\circ}$ compared to the normal of the sample surface. The polarization angle is measured with respect to the incident plane. S-polarization (blue) is defined as $0^{\circ}$ while P-polarization (green) is defined as $90^{\circ}$. c Pump laser polarization dependent time-resolved reflectivity in $\mathrm{Fe}_{3} \mathrm{GeTe}_{2}$ flake. The dashed line serves as the eye guide to show the oscillation peak shift in the spectra. $\mathbf{d}$ The extracted phonon frequency as a function of polarization angle. The error bar is based on numerical fitting.

Here, we apply time-resolved pump-probe technique to generate and study coherent phonons in exfoliated vdW Fe $\mathrm{GeTe}_{2}$ flakes. The experimental results and theoretical calculations confirm that the observed oscillation in reflectivity is attributed to the $\mathrm{A}_{1 \mathrm{~g}}$ phonon mode $(\sim 3.5 \mathrm{THz})$. In order to take advantage of the heavily asymmetric in-plane and out-of-plane interactions in vdW layer, we launch the pump laser beam at a large angle $\left(70^{\circ}\right)$ with respect to the normal to the vdW layer which enables out-ofplane electric field component during excitation. Surprisingly, we observe around 3\% phonon frequency modulation $(\sim 100 \mathrm{GHz})$ for the phonon mode generated by in-plane and out-of-plane polarized pump pulses. The phenomenon is reproducible in multiple flakes. The power dependent measurements are also carried out with fixed laser polarization and no obvious frequency modulation is observed which indicates a non-thermal origin of such phonon frequency control. We attribute the observed phonon frequency modulation to the modulation of restoring force of the lattice vibration in $\mathrm{Fe}_{3} \mathrm{GeTe}_{2}$ based on the orientation of laser polarization. Our findings contribute to the understanding of coherent phonon dynamics in vdW materials and the development of high speed phononic devices.

\section{RESULTS}

\section{Morphology and polarization dependent transient reflectivity of $\mathrm{Fe}_{3} \mathrm{GeTe}_{2}$ flake}

The morphology of the exfoliated $\mathrm{Fe}_{3} \mathrm{GeTe}_{2}$ flake is mapped out by Atomic Force Microscope (AFM) as shown in Fig. 1(a) (The AFM image for the other sample is shown in the supporting information). The size is about $4 \times 5 \mathrm{um}^{2}$, while the thickness is around $35 \mathrm{~nm}$ (about 44 layers), see inset of Fig. 1(a). The timeresolved pump-probe reflectivity measurements have been applied to vdW materials ${ }^{43,44}$. The setup splits the femtosecond pulsed laser beam $(800 \mathrm{~nm})$ from a Ti-Sapphire oscillator into two beams. One is used as pump beam and the other is called probe beam. Both beams are focused on the sample at the same spot. We use the femtosecond laser pulse (50 fs) in the pump beam to generate excitations and monitor the reflectivity of probe beam by varying the time of the probe pulse arriving the sample spot. In the conventional time-resolved pump-probe reflectivity measurements, the pump beam is incident perpendicular to the sample surface, which limits the orientation of pump pulse electric field only in the sample plane. In our experiments, we enable the outof-plane electric field component in the pump pulses by tilting the incident pump beam to investigate the effect of the electric field orientation on the coherent phonon excitation and relaxation in the transient reflectivity spectra. As shown in Fig. 1(b), the pump beam is sent into the sample about at $70^{\circ}$ angle with respect to the normal to the sample surface. We define the S-polarization as $0^{\circ}$ (in blue) and P-polarization as $90^{\circ}$ (in green). Thus $0^{\circ}$ polarization has only in-plane electric field component while $90^{\circ}$ polarization can exert significant out-of-plane electric field to the sample. See Supplementary Fig. 1 in the supplementary information for further description about the experimental setup.

The transient reflectivity spectra are plotted in Fig. 1(c) as a function of pump laser polarization (probe is S-polarized throughout the experiments). All the measurements were conducted under ambient temperature. The spectra in Fig. 1(c) start with strong fluctuations around delay time $t=0$ which represent the initial excitation of hot carriers when the pump 

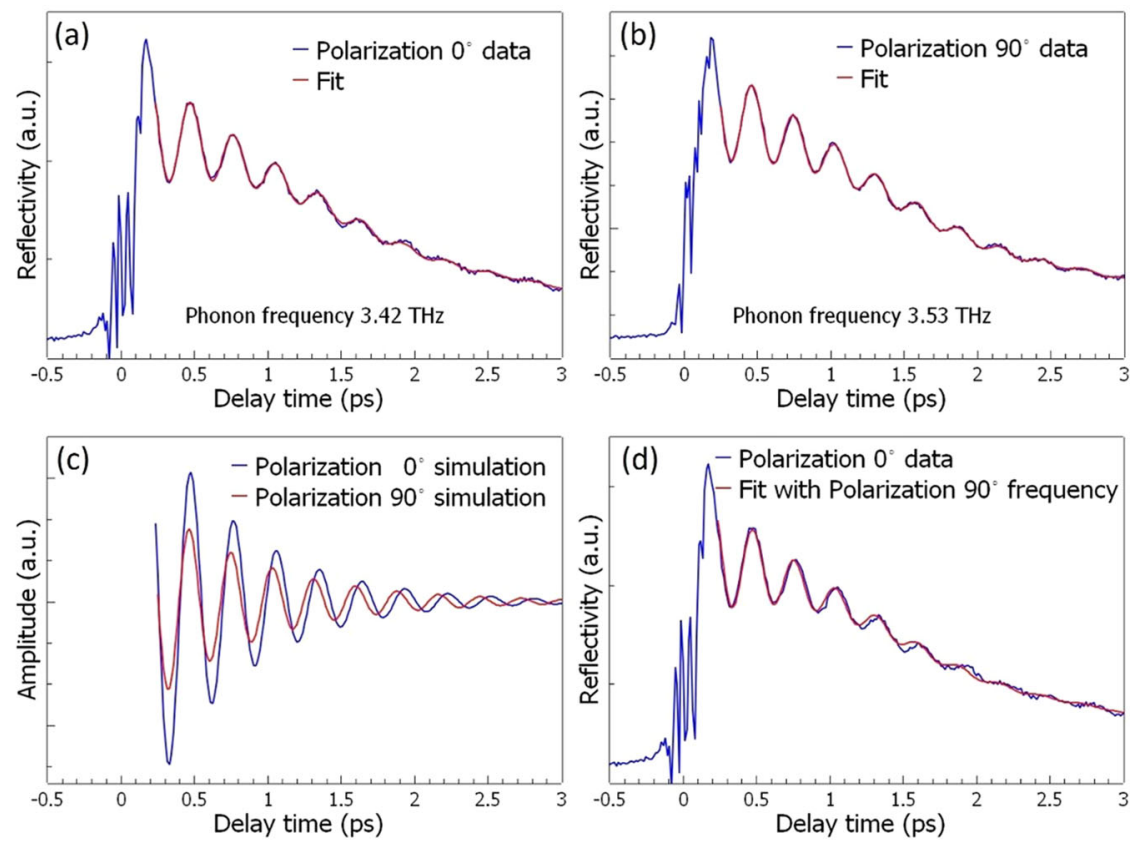

Fig. 2 Comparison of phonon frequencies at $0^{\circ}$ and $90^{\circ}$ laser polarizations. The transient reflectivity spectra under $0^{\circ}(\mathbf{a})$ and $90^{\circ}(\mathbf{b})$ pump polarizations. c Comparison of the simulated coherent phonon oscillation under $0^{\circ}$ and $90^{\circ}$ laser polarizations. d Numerical fitting of transient reflectivity data under $0^{\circ}$ laser polarization with coherent phonon frequency extracted from $90^{\circ}$ polarization case. The coherent phonon frequency different under two different pump polarizations is unambiguous.

pulse just arrives the sample followed by superposition of a periodical oscillation signal and exponential decay signal. The oscillation signal is attributed to the coherent phonon excitation in the sample ${ }^{45}$. The large exponential decay background is due to the hot carrier relaxation through lattice. Intriguingly, as the pump polarization is rotated from $0^{\circ}$ (in-plane) to $90^{\circ}$ (out-of-plane), we notice a small shift in the oscillation peak/valley positions which indicates a slightly altered coherent phonon frequency as a function of pump polarizations (see the dashed line in Fig. 1(c)). To further confirm it, we did numerical fittings to each transient reflectivity spectrum. The transient reflectivity signal can be approximated mathematically by a summation of exponential decay function and a damping oscillation function:

$\frac{\Delta R}{R} \sim A_{\mathrm{c}} \exp \frac{t}{\tau_{\mathrm{c}}}+A_{\mathrm{p}} \exp \frac{t}{\tau_{\mathrm{p}}} \cdot \cos \left(2 \pi f_{\mathrm{p}} * t+\varphi\right)+B$

where $A_{c}$ and $\tau_{c}$ are the amplitude and relaxation time for hot carrier excitation corresponding to the large decay background in the signal. $A_{\mathrm{p}}, \tau_{\mathrm{p}}$ and $f_{\mathrm{p}}$ are amplitude, lifetime, and frequency of the coherent phonon, respectively and $\varphi$ is the oscillation phase angle. The extracted coherent phonon frequency as a function of pump laser polarization given by Eq. (1) is plotted in Fig. 1(d). It turns out that the frequency of the coherent phonon is indeed modulated by the laser polarization. The phonon frequency monotonically increases when the pump polarization is varied from in-plane $\left(0^{\circ}\right)$ to out-of-plane direction $\left(90^{\circ}\right)$. The modulation of phonon frequency by the orientation of the pump polarization by $\sim 3 \%(100 \mathrm{GHz})$ is remarkable since the thermal control of similar phonon frequency change would require a temperature change of $\sim 360 \mathrm{~K}^{44}$. Furthermore, our result shows the ability to continuously control the phonon frequency which has interesting potential applications for high frequency phononic circuit/devices.

Figure 2(a) displays a fit of transient reflectivity data under pump polarization $0^{\circ}$ to Eq. (1). The coherent phonon frequency is extracted as $3.42 \pm 0.02 \mathrm{THz}$. The fit to transient reflectivity data under $90^{\circ}$ pump polarization in Fig. 2(b) gives a coherent phonon frequency of $3.53 \pm 0.02 \mathrm{THz}$. There is very good agreement between the numerical fitting curves (red solid lines) and the experimental data in both cases. The obtained phonon frequency shift is about $3 \%(\sim 100 \mathrm{GHz})$ for the two different pump polarization configurations, which is consistent with the observation in Fig. 1(c) and (d). We further plot the simulated phonon oscillations in transient reflectivity without the decaying background as shown in Fig. 2(c). It is obvious that the mismatch of oscillation peak and valley positions becomes more and more significant upon increasing the delay time, which indicates that the coherent phonon frequency is different in the two pump polarization configurations. This is exactly what happens when we use phonon frequency numerically extracted from $90^{\circ}$ pump polarization configurations to fit the $0^{\circ}$ data as shown in Fig. 2(d). We see that the discrepancy between the numerical fit (red solid line) and experimental data (blue solid line) becomes larger and larger in the longer time domain (e.g., 1-3 ps). Such laser polarization control of coherent phonon frequency is repeatable on different samples in our experiments (see Supplementary Fig. 2 in the supplementary information). The coherent phonon frequency is always higher (by about $3 \%$ ) in the pump polarization $90^{\circ}$ configuration than the $0^{\circ}$ configuration. In other words, when the pump polarization is in the sample plane, the excited coherent phonon frequency is relatively low, while it increases about $100 \mathrm{GHz}$ when the pump polarization becomes out of sample plane. These results and AFM images of additional samples can be found in the supporting information. In addition, to prove that the observed frequency modulation by laser polarization is indeed because of 2D vdW material (with the in-plane/out-of-plane) structural anisotropy, we also did the same pump polarization dependent transient reflectivity experiments with the same pump and probe incident configuration on a $2 \mathrm{~nm} \mathrm{CoFeB}$ thin film sample, and the spectra are shown in the supporting information (Supplementary Fig. 3 in the supplementary information). The transient reflectivity spectra of non-vdW thin films show clear coherent phonon oscillations with different modes under both pump polarizations. It is clear that the oscillation peak and valley positions for two different pump polarizations match well with each other which proves no frequency modulation/control by the pump laser polarization in non-vdW films. Our transient reflectivity 

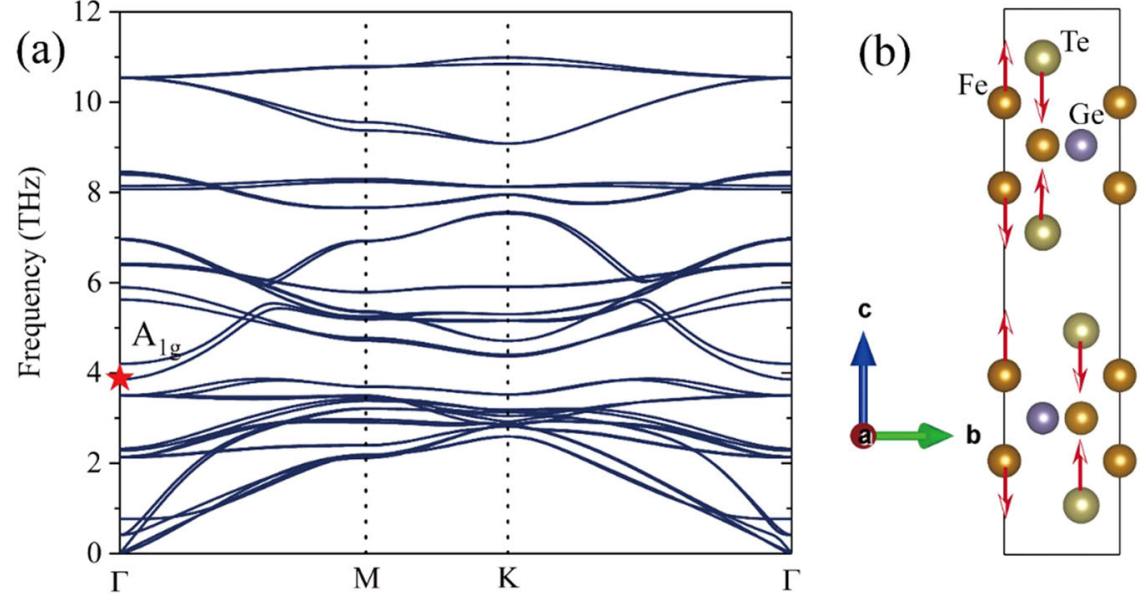

Fig. 3 First-principles calculation of phonon band structure of bulk $\mathrm{Fe}_{3} \mathrm{GeTe}_{2}$. a The calculated phonon dispersion of layered Fe $\mathrm{TeTe}_{2}$. The red star indicates the phonon mode branch $A_{1 g}$ observed in our experiment. (Other phonon modes such as $A_{2 u}, B_{1 g}, B_{2 u}, E_{2 g}, E_{1 u}, E_{1 g}$ are not shown in the figure for brevity). $\mathbf{b}$ The schematic of the $A_{1}$ vibrational mode in $\mathrm{Fe}_{3} \mathrm{GeTe}_{2}$, where the relative vibration direction of the atoms is labeled by the arrows.

spectrum on substrate (Supplementary Fig. 4 in the supplementary information) shows no oscillatory signal, which confirms that the transient reflectivity signal from substrate does not contribute to the coherent phonon oscillation signal.

\section{First-principles calculations}

To understand the observed laser polarization control of coherent phonon frequency in $\mathrm{Fe}_{3} \mathrm{GeTe}_{2}$, we calculated the phonon band structures of bulk $\mathrm{Fe}_{3} \mathrm{GeTe}_{2}$ by density functional theory (DFT). The phonon dispersion of $\mathrm{Fe}_{3} \mathrm{GeTe}_{2}$ can be observed in Fig. 3(a). The high symmetry path is $\Gamma-\mathrm{M}-\mathrm{K}-\Gamma$, the longitudinal acoustic (LA) and transverse acoustic (TA) branches correspond to vibrations within the plane, and the other mode (ZA) corresponds to out-of-plane vibration. It is worth mentioning that there is no imaginary frequency near the $\Gamma$ point, which indicates that the structure is thermodynamically stable.

The crystal structure of $\mathrm{Fe}_{3} \mathrm{GeTe}_{2}$ is p63/MMC space group [see Fig. $3(b)]$ with the $A_{1 g}$ mechanical representations. The Fe and $\mathrm{Ge}$ atoms located at $4 \mathrm{e}$ and $4 \mathrm{f}$ Wyckoff positions compose the $A_{1 g}$ Raman active modes. To be specific, 4 Fe atoms at the 4 e Wyckoff position vibrate in the out-of-plane direction, which is the same for $4 \mathrm{Te}$ atoms at the $4 \mathrm{f}$ Wyckoff position [see Fig. 3(b)]. We also calculated the vibration eigenvector of the Raman active $A_{1 g}$ mode. The phonon frequency of this $A_{1 g}$ vibrational mode is $3.86 \mathrm{THz}$, which is labeled as a red star in the phonon dispersion in Fig. 3(a). Note that the phonon frequency obtained in our experiments is around $3.50 \mathrm{THz}$, which is a little off from the calculated value. The discrepancy can be attributed to (1) the firstprinciples calculations are carried out at absolute zero temperature, but as the temperature increases, the phonon frequency normally decreases due to lattice expansion ${ }^{44,46}$, and (2) sample size effect: the first-principles calculations are carried out for bulk crystal, while experiments are conducted on nano-films or finite thickness. Raman spectroscopy is an important experimental method to characterize solid phonon physics, which reflects the magnitude of the quantized energy of lattice vibration. The vibrational frequency of the $A_{1 g}$ mode obtained by Raman spectroscopy in $\mathrm{Fe}_{3} \mathrm{GeTe}_{2}$ flakes at room temperature and with similar sample size is in great agreement with our experimental observations ${ }^{46}$. The origin of the phonon is lattice vibration in which the interactions between atoms (restoring forces) essentially determine the vibration frequencies. It has been reported that, in 2D materials (e.g., $M_{0} S_{2}$ ), the frequency of $A_{1} g$ phonon mode shows redshift with a decreasing number of layers which is attributed to the weakening of the restoring forces acting upon vibrations while the $E_{2 g}$ phonon mode blueshifts ${ }^{47-50}$. It is known that the vibration of atoms in $A_{1 g}$ phonon mode is out-of-plane (refer to monolayer plane) while that of $E_{2 g}$ phonon mode is inplane. The opposite response of phonon frequency for $A_{1} g$ and $\mathrm{E}_{2} \mathrm{~g}$ mode in $2 \mathrm{D}$ materials may root in the heavily asymmetric inplane/out-of-plane inter-atomic interactions and suggests the possibility to control the phonon frequency by instantaneous modification of restoring forces, e.g., through laser polarization.

To obtain further insight into the phenomenon, we calculated the electronic charge distribution for different atoms in $\mathrm{Fe}_{3} \mathrm{GeTe}_{2}$ lattice as shown in Fig. 4(a). It can be shown that electron distributions around $\mathrm{Fe}, \mathrm{Ge}$, and Te atoms show different levels of overlap between each other which qualitatively represent the interaction between neighboring atoms. A comparison of the electronic charge distribution overlap along in-plane orientation ( $a$ and $b$ axes) and out-of-plane orientation ( $c$-axis) shows that the electron distribution overlap between Fe-Fe and Te-Ge-Te along the out-of-plane orientation is much stronger than that along the in-plane orientation. This agrees with the fact that the highly symmetric in-plane/out-of-plane interactions exist in $\mathrm{Fe}_{3} \mathrm{GeTe}_{2}$ lattice and potentially can be selectively excited by the laser pulse with different polarization orientations. It has been reported that the ultrafast laser pulses can induce transient superconductivity up to room temperature in $\mathrm{YBa}_{2} \mathrm{Cu}_{3} \mathrm{O}_{6+\mathrm{x}}{ }^{51,52}$. Later femtosecond $X$-ray diffraction experiments showed that the ultrafast lattice excitation induces modification in the $\mathrm{Cu}-\mathrm{O}_{2}$ intra-bilayer and inter-bilayer distances, accompanied by anisotropic changes in the in-plane $\mathrm{O}-\mathrm{Cu}-\mathrm{O}$ bond buckling ${ }^{53}$. In 2D materials, the coherent light-matter interaction that can control the symmetry breaking in $\mathrm{WS}_{2}{ }^{54,55}$, WSe ${ }_{2}^{56,57}$, and symmetry switching in $\mathrm{ZrTe}_{5}{ }^{58}$ has been observed $^{59}$. The ultrafast phase transition in $2 \mathrm{D} \mathrm{VSe}{ }_{2}$ has also been reported and attributed to electron-lattice interaction ${ }^{60}$. Most recently, Afanasiev et.al., has demonstrated non-thermal lattice control through mid-infrared electric field pulses driven phonons to manipulate the magnetic interactions in $\mathrm{DyFeO}_{3}{ }^{61}$. Moreover, the photoinduced magnetic anisotropy in two-dimensional van der Waals (vdW) antiferromagnet $\mathrm{NiPS}_{3}$ shows the control of the magnon amplitude by pump laser polarization ${ }^{62}$. In our case, the polarization of femtosecond laser pulse essentially represents the direction of few-cycle oscillating electric field with super high frequency. In our experimental configuration, the pump laser is incident with large tilted angle which provides maneuverable inplane and out-of-plane polarization/electric field components. It is possible that the laser pulses with different polarizations are 

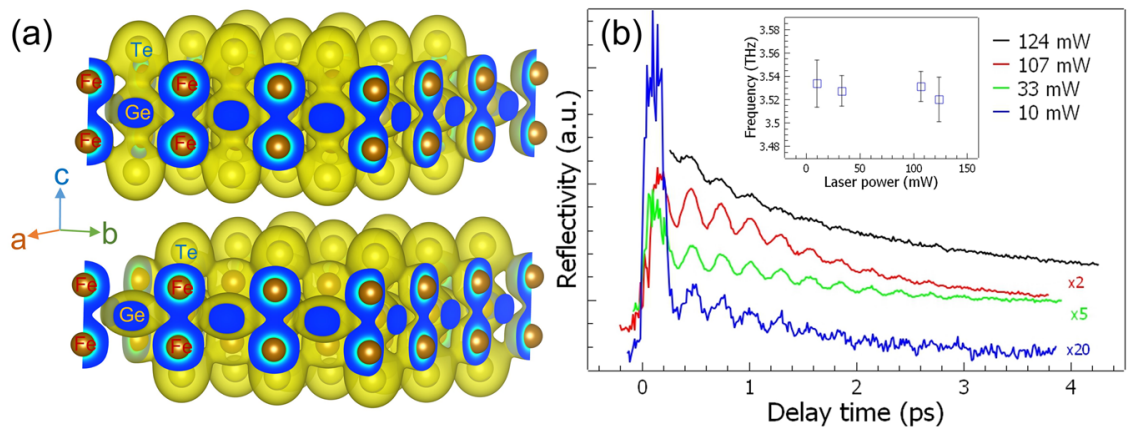

Fig. 4 Calculated electronic charge distribution and pump power dependent transient reflectivity spectra. a Calculated electronic charge distribution between different atoms. b Pump laser power dependence of transient reflectivity spectra under $90^{\circ}$ pump polarization. Each of these spectra is averaged over 40 times. The scale of each spectrum is adjusted to fit in one figure frame since they are measured with different sensitivity. The inset of (b) shows the extracted frequency of each spectrum.

coupled to the anisotropic electron-lattice interaction in $\mathrm{Fe}_{3} \mathrm{GeTe}_{2}$ asymmetrically (in-plane/out-of-plane) and modify the restoring force for $A_{1 g}$ phonon mode, which in turn induce the phonon frequency modulation.

\section{Laser power dependent transient reflectivity of Fe3GeTe2 flake}

In addition, we conducted pump power dependent transient reflectivity measurements with a fixed pump polarization $\left(90^{\circ}\right)$ to further understand the origin of the observed coherent phonon frequency control. Based on previous reports ${ }^{44}$, the phonon frequency varies slightly with temperature which may be due to the thermal expansion of the lattice. Thus, one of the hypotheses for the phonon frequency modulation is based on the thermal origin in which the absorption of pump pulse energy under two orthogonal pump polarizations is significantly different causing substantial local temperature difference, in turn changing the phonon frequency. In transient reflectivity experiments, pump laser power is usually proportional to the local temperature on the sample surface. If the thermal effect is the major contribution, the pump laser power should also be able to tune the phonon frequency. Figure 4(b) shows the transient reflectivity spectra under $90^{\circ}$ pump polarization at different pump laser powers. Under low pump laser power (e.g., $10 \mathrm{~mW}$ ), the coherent phonon excitation is very weak so the oscillations in the spectrum are rough even after averaging over 40 times. Under high pump laser power (e.g., $124 \mathrm{~mW}$ ), the oscillations are clear, but the lifetime is very short compared to those measured under intermediate pump laser powers (e.g., $33 \mathrm{~mW}$ and $107 \mathrm{~mW}$ ). This is attributed to the thermal effect induced phonon scattering that significantly shortens the coherent phonon lifetime, which indicates comparatively high local temperature in a $5 \mu \mathrm{m}$ size flake when compared to, for example, $33 \mathrm{~mW}$ case. However, the oscillation peak and valley positions for all four spectra match with each other very well, which shows almost no frequency modulation by pump power within our achievable range. Hence, the thermal effect is unlikely the major contribution to the observed pump polarization control of coherent phonon frequency.

\section{DISCUSSION}

In summary, we report an efficient control of coherent phonons in $\mathrm{Fe}_{3} \mathrm{GeTe}_{2}$ by changing laser polarizations. Unlike the conventional time-resolved pump-probe experiments, our pump laser beam used a very large angle of incidence $\left(70^{\circ}\right)$ with respect to the sample plane which enables out-of-plane electric field component when rotating the laser polarizations. A wide-range phonon frequency modulation by about $3 \%(100 \mathrm{GHz})$ was observed. Our firstprinciples calculations reveal asymmetric in-plane and out-of-plane inter-atomic interactions in $\mathrm{Fe}_{3} \mathrm{GeTe}_{2}$ allow the laser pulse with different polarization orientations to couple to the anisotropic electron-lattice interactions and modify the vibration stiffness/ restoring force of $A_{1 g}$ phonon mode asymmetrically. Moreover, the pump power dependent transient reflectivity results show no obvious coherent phonon frequency modulation confirming a nonthermal origin for such manipulation. Our findings not only provide the fundamental understanding of the coherent phonon dynamics in layered materials but also exhibit the significance of phonon frequency modulations for the development of high frequency phononic devices.

\section{METHODS}

\section{Sample preparation}

The bulk $\mathrm{Fe}_{3} \mathrm{GeTe}_{2}$ single crystals were synthesized by chemical vapor transport method, and $\mathrm{Fe}_{3} \mathrm{GeTe}_{2}$ flakes were mechanically exfoliated and deposited on $260 \mathrm{~nm}$-thick-SiO $/ 2 / \mathrm{Si}$ chips.

\section{Transient reflectivity measurements}

The transient reflectivity results were collected by time-resolved pumpprobe spectroscopy. The pump beam was modulated by a mechanical chopper at $1.5 \mathrm{kHz}$. The probe beam was sent into an objective (perpendicular to the sample surface) to be focused onto micrometersize samples. The probe reflectivity as a function of time delay between pump and probe pulses was collected by the same objective and read by a balanced detector. The transient reflectivity change was eventually determined and output by a lock-in amplifier.

\section{Density functional theory}

Our first-principles calculations are based on DFT using the projector augmented wave method as implemented in the Vienna ab initio simulation package ${ }^{63-65}$. The second-order interatomic force constants were calculated by the finite displacement method and the phonon dispersion was then obtained by using the Phonopy package.

\section{DATA AVALABILITY}

The data that support the findings of this study are available from the corresponding author upon reasonable request.

Received: 19 June 2021; Accepted: 30 November 2021; Published online: 08 February 2022

\section{REFERENCES}

1. McNeil, R. et al. On-demand single-electron transfer between distant quantum dots. Nature 477, 439-442 (2011).

2. Chen, J. et al. Enhanced electron-phonon coupling for a semiconductor charge qubit in a surface phonon cavity. Sci. Rep. 5, 15176 (2015). 
3. Gustafsson, M. et al. Propagating phonons coupled to an artificial atom. Science 346, 207-211 (2014).

4. Satzinger, K. et al. Quantum control of surface acoustic-wave phonons. Nature 563, 661-665 (2018).

5. Bienfait, A. et al. Phonon-mediated quantum state transfer and remote qubit entanglement. Science 364, 368-371 (2019).

6. Fuhrmann, D. et al. Dynamic modulation of photonic crystal nanocavities using gigahertz acoustic phonons. Nat. Photon 5, 605-609 (2011).

7. Li, H., Tadesse, S., Liu, Q. \& Li, M. Nanophotonic cavity optomechanics with propagating acoustic waves at frequencies up to $12 \mathrm{GHz}$. Optica 2, 826-831 (2015).

8. Tadesse, S., Li, H., Liu, Q. \& Li, M. Acousto-optic modulation of a photonic crystal nanocavity with Lamb waves in microwave K band. Appl. Phys. Lett. 107, 201113 (2015).

9. Tadesse, S. \& Li, M. Sub-optical wavelength acoustic wave modulation of integrated photonic resonators at microwave frequencies. Nat. Commun. 5, 5402 (2014).

10. Young, E. et al. Subterahertz acoustical pumping of electronic charge in a resonant tunneling device. Phys. Rev. Lett. 108, 226601 (2012).

11. Moss, D. et al. Ultrafast strain-induced current in a GaAs schottky diode. Phys. Rev. Lett. 106, 066602 (2011).

12. Lanzillotti-Kimura, N. et al. Coherent control of sub-terahertz confined acoustic nanowaves: Theory and experiments. Phys. Rev. B 84, 115453 (2011).

13. Li, N. et al. Colloquium: Phononics: Manipulating heat flow with electronic analogs and beyond. Rev. Mod. Phys. 84, 1045-1066 (2012).

14. Wang, L. \& Hu, S. Photoacoustic tomography: In vivo imaging from organelles to organs. Science 335, 1458-1462 (2012).

15. Safavi-Naeini, A., Van Thourhout, D., Baets, R. \& Van Laer, R. Controlling phonons and photons at the wavelength scale: integrated photonics meets integrated phononics. Optica 6, 213-232 (2019).

16. Das, A. et al. Monitoring dopants by Raman scattering in an electrochemically top-gated graphene transistor. Nat. Nanotechnol. 3, 210-215 (2008).

17. Zhao, Y. et al. Observation of logarithmic Kohn anomaly in monolayer graphene. Phys. Rev. B 102, 165415 (2020).

18. Conley, H. J. et al. Bandgap engineering of strained monolayer and bilayer $\mathrm{MoS}_{2}$. Nano Lett. 13, 3626-3630 (2013).

19. Zhu, C. R. et al. Strain tuning of optical emission energy and polarization in monolayer and bilayer MoS 2 . Phys. Rev. B 88, 121301(R) (2013).

20. Lee, C. et al. Anomalous lattice vibrations of single- and few-layer $\mathrm{MoS}_{2}$. Acs Nano 4, 2695-2700 (2010).

21. Velicky, M. \& Toth, P. From two-dimensional materials to their heterostructures: an electrochemist's perspective. Appl. Mater. Today 8, 68-103 (2017).

22. Lanzillo, N. et al. Temperature-dependent phonon shifts in monolayer $\mathrm{MoS}_{2}$. Appl. Phys. Lett. 103, 093102 (2013).

23. Taube, A. et al. Temperature-dependent nonlinear phonon shifts in a supported $\mathrm{MoS}_{2}$ monolayer. ACS Appl. Mater. Interfaces 6, 8959-8963 (2014).

24. Geim, A. \& Grigorieva, I. Van der Waals heterostructures. Nature 499, 419-425 (2013).

25. Wang, Q. et al. Electronics and optoelectronics of two-dimensional transition metal dichalcogenides. Nat. Nanotechnol. 7, 699-712 (2012).

26. Butler, S. et al. Progress, challenges, and opportunities in two-dimensional materials beyond graphene. Acs Nano 7, 2898-2926 (2013).

27. Hung, N., Nugraha, A. \& Saito, R. Two-dimensional InSe as a potential thermoelectric material. Appl. Phys. Lett. 111, 092107 (2017).

28. O'Connell, A. et al. Quantum ground state and single-phonon control of a mechanical resonator. Nature 464, 697-703 (2010).

29. Armstrong, M. et al. Observation of terahertz radiation coherently generated by acoustic waves. Nat. Phys. 5, 285-288 (2009).

30. Heywood, S. et al. Heterodyne mixing of millimetre electromagnetic waves and sub-THz sound in a semiconductor device. Sci. Rep. 6, 30396 (2016).

31. Wehmeier, L. et al. Polarization-dependent near-field phonon nanoscopy of oxides: $\mathrm{SrTiO}_{3}, \mathrm{LiNbO}_{3}$, and $\mathrm{PbZr}_{0.2} \mathrm{Ti}_{0.8} \mathrm{O}_{3}$. Phys. Rev. B 100, 035444 (2019).

32. Duong, D., Yun, S. \& Lee, Y. van der Waals layered materials: opportunities and challenges. Acs Nano 11, 11803-11830 (2017).

33. Fei, Z. et al. Two-dimensional itinerant ferromagnetism in atomically thin $\mathrm{Fe}_{3} \mathrm{GeTe}_{2}$. Nat. Mater. 17, 778-782 (2018).

34. Gong, C. \& Zhang, X. Two-dimensional magnetic crystals and emergent heterostructure devices. Science 363, eaav4450 (2019).

35. Parka, I. K., Gong, C., Kim, K. \& Lee, G. Controlling interlayer magnetic coupling in the two-dimensional magnet $\mathrm{Fe}_{3} \mathrm{GeTe}_{2}$. Phys. Rev. B 105, 014406 (2022).

36. Gong, C. et al. Discovery of intrinsic ferromagnetism in two-dimensional van der Waals crystals. Nature 546, 265-269 (2017).

37. Kim, K. et al. Large anomalous Hall current induced by topological nodal lines in a ferromagnetic van der Waals semimetal. Nat. Mater. 17, 794-799 (2018).

38. Wang, Z. et al. Tunneling Spin Valves Based on $\mathrm{Fe}_{3} \mathrm{GeTe}_{2} / \mathrm{hBN} / \mathrm{Fe}_{3} \mathrm{GeTe}_{2}$ van der Waals Heterostructures. Nano Lett. 18, 4303-4308 (2018).
39. Zhang, Y. et al. Emergence of Kondo lattice behavior in a van der Waals itinerant ferromagnet, $\mathrm{Fe}_{3} \mathrm{GeTe}_{2}$. Sci. Adv. 4, eaao6791 (2018).

40. Li, Q. et al. Patterning-induced ferromagnetism of $\mathrm{Fe}_{3} \mathrm{GeTe}_{2}$ van der Waals materials beyond room temperature. Nano Lett. 18, 5974-5980 (2018).

41. Tu, Z. et al. Ambient effect on the Curie temperatures and magnetic domains in metallic two-dimensional magnets. npj 2D Mater. Appl. 5, 62 (2021).

42. Zhu, J. et al. Electronic correlation and magnetism in the ferromagnetic metal $\mathrm{Fe}_{3} \mathrm{GeTe}_{2}$. Phys. Rev. B 93, 144404 (2016).

43. Jin, C. et al. Ultrafast dynamics in van der Waals heterostructures. Nat. Nanotechnol. 13, 994-1003 (2018).

44. Guo, J. et al. Enhanced coherent phonon excitation in $\mathrm{Fe}_{3} \mathrm{GeTe}_{2}$ via resonance Raman effect. Phys. Rev. B 103, 024302 (2021).

45. Miao, X. et al. Layer-dependent ultrafast carrier and coherent phonon dynamics in black phosphorus. Nano Lett. 18, 3053-3059 (2018).

46. Du, L. et al. Lattice dynamics, phonon chirality, and spin-phonon coupling in 2D itinerant ferromagnet $\mathrm{Fe}_{3} \mathrm{GeTe}_{2}$. Adv. Funct. Mater. 29, 1904734 (2019).

47. Berkdemir, A. et al. Identification of individual and few layers of $\mathrm{WS}_{2}$ using Raman Spectroscopy. Sci. Rep. 3, 1755 (2013).

48. Yamamoto, M. et al. Strong enhancement of Raman scattering from a bulkInactive vibrational mode in few-layer MoTe 2 . Acs Nano 8, 3895-3903 (2014).

49. Zhao, W. et al. Lattice dynamics in mono- and few-layer sheets of $\mathrm{WS}_{2}$ and $\mathrm{WSe}_{2}$. Nanoscale 5, 9677-9683 (2013).

50. Lu, X. et al. Rapid and nondestructive identification of polytypism and stacking sequences in few-layer Molybdenum Diselenide by Raman spectroscopy. Adv. Mater. 27, 4502-4508 (2015).

51. Kaiser, $S$. et al. Optically induced coherent transport far above $T_{C}$ in underdoped $\mathrm{YBa}_{2} \mathrm{Cu}_{3} \mathrm{O}_{6+\delta}$. Phys. Rev. B 89, 184516 (2014).

52. $\mathrm{Hu}, \mathrm{W}$. et al. Optically enhanced coherent transport in $\mathrm{YBa}_{2} \mathrm{Cu}_{3} \mathrm{O}_{6.5}$ by ultrafast redistribution of interlayer coupling. Nat. Mater. 13, 705-711 (2014).

53. Mankowsky, R. et al. Nonlinear lattice dynamics as a basis for enhanced superconductivity in $\mathrm{YBa}_{2} \mathrm{Cu}_{3} \mathrm{O}_{6.5}$. Nature 516, 71-73 (2014).

54. Sie, E. J. et al. Large, valley-exclusive Bloch-Siegert shift in monolayer $\mathrm{WS}_{2}$. Science 355, 1066-1069 (2017).

55. Sie, E. J. et al. Valley-selective optical Stark effect in monolayer WS $_{2}$. Nat. Mater. 14, 290-294 (2015).

56. Kim, J. et al. Ultrafast generation of pseudo-magnetic field for valley excitons in $\mathrm{WSe}_{2}$ monolayers. Science 346, 1205-1208 (2014).

57. Ye, Z., Sun, D. \& Heinz, T. F. Optical manipulation of valley pseudospin. Nat. Phys. 13, 26-29 (2017).

58. Luo, L. et al. A light-induced phononic symmetry switch and giant dissipationless topological photocurrent in $\mathrm{ZrTe}_{5}$. Nat. Mater. 20, 329-334 (2021).

59. Du, L. et al. Engineering symmetry breaking in 2D layered materials. Nat. Rev. Phys. 3, 193-206 (2021).

60. Biswas, D. et al. Ultrafast triggering of insulator-metal transition in twodimensional VSe $e_{2}$. Nano Lett. 21, 1968-1975 (2021).

61. Afanasiev, D. et al. Ultrafast control of magnetic interactions via light-driven phonons. Nat. Mater. 20, 607-611 (2021).

62. Afanasiev, D. et al. Controlling the anisotropy of a van der Waals antiferromagnet with light. Sci. Adv. 7, abf3096 (2021).

63. Kresse, G. \& Furthmüller, J. Efficiency of ab-initio total energy calculations for metals and semiconductors using a plane- wave basis set. Comput. Mater. Sci. 6, 15-50 (1996).

64. Kresse, G. \& Furthmüller, J. Efficient iterative schemes for ab initio total-energy calculations using a plane-wave basis set. Phys. Rev. B 54, 11169-11186 (1996).

65. Kresse, G. \& Joubert, D. From ultrasoft pseudopotentials to the projector augmented-wave method. Phys. Rev. B 59, 1758-1775 (1999).

\section{ACKNOWLEDGEMENTS}

Research reported in this publication was supported in part by the NSF and SC EPSCoR/IDeA Program under NSF Award \#OIA-1655740 and GEAR CRP 20-GC02, and NSF Award No. 2030128. The views, perspective, and content do not necessarily represent the official views of the SC EPSCoR Program nor those of the NSF. The work at Rutgers University was supported by the DOE under Grant No. DOE: DE-FG0207ER46382. C.G. acknowledges the grants from Northrop Grumman Mission Systems' University Research Program, Naval Air Warfare Center Aircraft Division, and Army Research Laboratory cooperative agreement number W911NF-19-2-0181.

\section{AUTHOR CONTRIBUTIONS}

Y.G. conceived the project and designed experiments. N.H. and J.K. conducted transient reflectivity measurements under Y.G.'s supervision. M.H. and Z.Y. carried out DFT calculations. T.X. conducted 2D sample exfoliation under C.G.'s supervision. A.A.T. and W.M. carried out AFM measurements and provided figures. X.X. synthesized bulk 
$\mathrm{Fe}_{3} \mathrm{GeTe}_{2}$ crystals under S.-W.C.'s supervision. N.K. and C.G. participated in the result discussion. Y.G. and C.G. wrote the paper with DFT calculation figures and paragraphs provided by M.H. and Z.Y. All authors approved the paper submission.

\section{COMPETING INTERESTS}

The authors declare no competing interests.

\section{ADDITIONAL INFORMATION}

Supplementary information The online version contains supplementary material available at https://doi.org/10.1038/s41699-021-00275-4.

Correspondence and requests for materials should be addressed to Yu Gong, Ming Hu or Cheng Gong.

Reprints and permission information is available at http://www.nature.com/ reprints
Publisher's note Springer Nature remains neutral with regard to jurisdictional claims in published maps and institutional affiliations.

(i) Open Access This article is licensed under a Creative Commons Attribution 4.0 International License, which permits use, sharing, adaptation, distribution and reproduction in any medium or format, as long as you give appropriate credit to the original author(s) and the source, provide a link to the Creative Commons license, and indicate if changes were made. The images or other third party material in this article are included in the article's Creative Commons license, unless indicated otherwise in a credit line to the material. If material is not included in the article's Creative Commons license and your intended use is not permitted by statutory regulation or exceeds the permitted use, you will need to obtain permission directly from the copyright holder. To view a copy of this license, visit http://creativecommons. org/licenses/by/4.0/.

(c) The Author(s) 2022 